\title{
Beyond sexual selection: The evolution of sex differences from brain to behavior
}

Sexual selection has received a great deal of attention in the last few decades (Owens, 2006), and continues to be a central topic of interest for behavioral ecologists. First, it is useful to go back to the historical definition of sexual selection:

when the males and the females of any animal have the same general habits of life, but differ in their structure, colour, or ornament, such differences have been mainly caused by sexual selection; that is, individual males have had, in successive generations, some slight advantage over other males, in their weapons, means of defence, or charms; and have transmitted these advantages to their male offspring.

Charles Darwin, On the Origin of Species by Means of Natural Selection, 1859 (chap. IV, pp. 89-90)

Slight differences in reproductive success may be caused by male weapons and/or charms, exaggerated sexual characteristics. These are the key words of The Descent of Man, and Selection in Relation to Sex (1871): sexual selection meant "ardent males and choosy females", i.e. male-male fighting and female choice. Work since Darwin's time has enlarged the theory to note that females can be aggressive and males choosy, depending on the circumstances. In addition, sexual selection is recognized to occur both before and after copulation. Despite early skepticism and "sexual selection amnesia" (West-Eberhard, in this issue), a growing body of research and recent models are now centered on this highly heuristic theory, making it one of the best studied areas of behavioral ecology alongside animal signals and host-parasite interactions (Fig. 1).

There is broad consensus that the occurrence of sexually dimorphic traits among animals - especially in males - often may be explained by sexual selection, but the processes by which sexual selection operate are still controversial. Exaggerated sexual signals have been considered indicators of "good genes" (Hamilton and Zuk, 1982); thus, males with exaggerated traits also sire offspring that have higher fitness. Sexual ornaments, if costly, may be honest cues of mate quality (Zahavi, 1975) and health (Zuk and Wedell, 2014), solving the conundrum of how heritable variance in fitness is maintained.

On the other hand, in accordance with the "aesthetic capacity" of females, the essence of Darwin's argument (1871), and the runaway co-evolution between male trait and female preference (Fisher, 1958), bizarre courtship displays or conspicuous fighting specializations may evolve because they are merely preferred, without no additional information. The female "taste for the beautiful" has been recently advanced as "Darwin's really dangerous idea” (Prum, 2012): male traits could be preferred, regardless of any additional quality. But Darwin was perhaps open to both these options, if females "will select those that are vigorous and well-armed, and in other respect the most attractive" (1871, chap. VIII, 262). If some traits may evolve as armaments and ornaments, with a dual function (Berglund et al., 1996), "runaway selection leads to good genes" (Chandler et al., 2013). Kokko et al. (2002) claimed that there is a continuum from the evolutionary mechanisms of good genes and female esthetic choice through the indirect benefit of fitter offspring; thus, the distinction between "arbitrary and non-arbitrary male traits is, in itself, arbitrary" (Kokko et al., 2006).

Given the challenging debate on sexual selection, it is no surprise that, over the last 30 years, research has focused on the evolution of mating systems and male armaments/ornaments, sexual signals as good-genes indicators, alternative reproductive tactics, sexual dimorphism from gametes to parental roles, and much more. But along with these well-researched topics, related or complementary

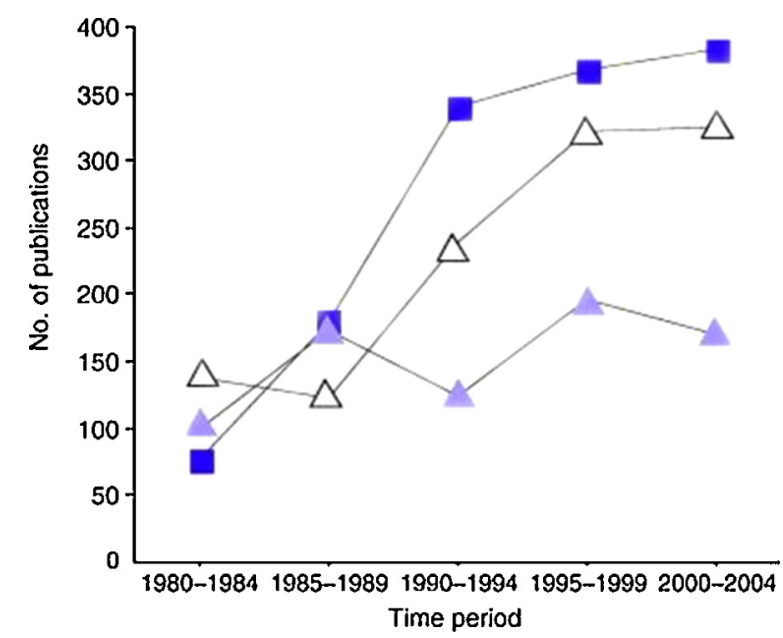

Fig. 1. The number of publications per 10,000 papers on sexual selection (closed squares), host-parasite interactions (open triangles), animal signals (closed triangles) published over the past 25 years in the journals Behavioral Ecology, Animal Behaviour and Behavioral Ecology and Sociobiology.

Source: From Owens (2006). 
ones may have been neglected or received much less attention: male rather than female mate choice; female-female competition; female promiscuity and cryptic choice; conflict and coevolution between the sexes in brain structure, gene expression and cognitive functions; the sensory trap hypothesis; the trade-off between immunity and mating efforts; and the apparent paradox of samesex behavior, among others. Often it is in these departures from the usual way of thinking that we gain new insights. In addition, a comparative approach and less-standard model systems may allow to better understand the evolutionary development of typical and atypical sexual behavior.

In this special issue, we explore the other side of sexual selection by viewing sexual differences at a variety of scales, ranging from neural pathways and individual personality to a macroevolutionary perspective. Sex differences in the brain have been well documented, but their adaptive value has been only superficially investigated. Sexual dimorphism may mediate learning abilities involved in mate choice and competition for partners: neuro-motor skills to display, advertisement song, partner's evaluation at a fine-scale, perceptive enhancement, sensory exploitation and so on. If this is the case, then such neural differences are sexually selected traits, like antlers and nuptial plumage, and they are best understood within the framework of sexual selection.

Neural sexual dimorphism is a particular topic of this Special Issue because the brain is a target of sexual selection. In the last chapter of The Descent of Man, Darwin underlines the evolutionary factors that open the way to the approach of comparative neuroanatomy.

Everyone who admits the principle of evolution, and yet feels great difficulties in admitting that female mammals, birds, reptiles, and fish, could have acquired the high standard of taste which is implied by the beauty of the males, and which generally coincides with our own standard, should reflect that in each member of the vertebrate series the nerve-cells of the brain are the direct offshoots of those possessed by the common progenitor of the whole group. It thus becomes intelligible that the brain and mental faculties should be capable under similar conditions of nearly the same course of development, and consequently of performing nearly the same functions.

Charles Darwin, The Descent of Man, and Selection in Relation to Sex, 1871 (chap. XXI, p. 401)

Brain sex differences involve developmental, ecological and taxonomic differences. "Birds and mammals exhibit highly canalized sexual differentiation, amphibians and reptiles are more plastic, and teleost fishes are extremely labile" (Francis, 1995). At least some of these differences might be best understood within the framework of sexual selection.

The introductory essay by West-Eberhard is mostly devoted to the coevolution of sensory-response systems under natural and sexual selection, better, under "social selection", of interest for neurobiological studies which put male-female differences and conflicts in an evolutionary perspective. Social competition exploits "a multitude of sensory-response capacities". As West-Eberhard emphasizes, "indicators of quality seem unlikely to account for the great complexity of courtship displays". Attractiveness may assume "a stimulatory value per se, above and beyond any significance they may have as signs of good genes", better, "good survival genes". The evolution of multimodal signals and extravagant male phenotypes "exceeds that expected under sexual section": a process, for the author, clearly distinct from natural selection, although both mechanisms may mutually reinforce and "interplay in signal evolution". Consistent with a co-evolutionary process driving complex neural and behavioral-morphological traits, West-Eberhard may conclude that "a spectacular product of natural-social selection is the human brain", in line with the Darwinian theory.

Herein we present a series of basic research and review papers relevant for understanding sexual selection and its implications in behavioral, physiological and neuro-endocrine studies, organized in three sections: first, male extreme behavioral traits as suitable model systems to investigate hormonal and neural bases of exorbitant mating efforts; second, apparently maladaptive behavior and exceptions to standard sexual selection; third, cognitive neurobiology and sex-related differences in brain and behavior in humans and other primates.

\section{Male life-history: "Live hard, die young”}

The cost of reproduction in various forms is well documented (Reznick, 1985). In many species, this cost is manifested as a reduction in longevity or immune response in males (Zuk and McKean, 1996). In the case of pigmy rattlesnakes, this tradeoff was summarized as "Live fast, love hard and die young" (May et al., 1997). A recent paper shows that the perception of female sexual pheromones is sufficient to limit the lifespan of male fruit flies (Gendron et al., 2014). Male sacrifice and autotomy of signaling appendages in spiders offer a good example of high mating investment (Herberstein et al., in this issue). Sexual selection has shaped male courtship to honestly communicate the identity and the quality of a potential mate and thus to reduce the risk of predation by the female. The trade-off between extreme sexual dimorphism and lower rates of cannibalism across species suggests that the evolution of complexity may inhibit female attack. Multimodal signaling, including visual, chemical, but also vibrational components, implies considerable capacity to emit, process and respond to such elaborate messages, although a few neurobiological studies explore in detail the role of the central and peripheral nervous systems in integrating these sexual signals, from both a female and a male perspective.

Multimodal signaling is surely involved in the leks - i.e. the nuptial arenas - of paper wasps, where hundreds of males display at landmarks (Beani et al., in this issue), as well as in the expanded lek mating system of manakins, performing their lonely dances in the neotropical forest (Fusani et al., in this issue). Male hymenopterans have neither armaments nor ornaments, and are ephemeral and elusive, difficult to observe in comparison to the impressive architecture of the colonies - a feminine world. The impact of sexual selection on the evolution of the social insect societies is a subject that is slowly developing, such as the subtle role of males and the cognitive bases of their behavior, from courtship to alternative mating tactics, selective mate choice and sperm allocation. One can view the male neglected phenotype in wasps, bees and ants as "a natural experiment to compare the drives of natural versus sexual selection, parental versus mating efforts, and their associated neurogenomic mechanisms" (Beani et al., in this issue).

The bizarre acrobatic performance of the Golden-collared manakin, the focus of long-term research (Fusani et al., in this issue), has been interpreted as an honest indicator of male motor skills, which are influenced - in their organization and activation - by gonadal hormones, expressed in a sex-dimorphic way in the brain, spinal cord and skeletal muscles: "an androgen-dependent phenotype", although this neural circuitry is surprisingly present - but not active - in females (Fusani et al., in this issue). The female, equipped with superb visual processing, may assess and compare, at a fine scale, the male performance, driving the evolution of this complex courtship, which implies a rigid choreography of the nuptial arena (Barske et al., 2011). “On the whole, birds appear to be the most aesthetic of all animals, excepting of course man, and they have nearly the same taste for the beautiful as we have" (Darwin, 1871, chap. XI, 
p. 466). As outlined by the authors, the esthetic value of male performance, charming females through novelty and diversity, does not exclude the genetic quality of male performance. Again, runaway selection and good genes benefits could be viewed as interactive synergic mechanisms (Chandler et al., 2013).

Songbirds offer a further example of species-specific neuromuscular systems controlling sexual displays, in this case the male vocal performance. The song of the Bengalese finch has been defined as "a window on the behavioral neurobiology of birdsong syntax" (Okanoya, 2004). The song of this species, domesticated in Japan for 250 years, is more complex and plastic than the stereotyped simple song of the wild ancestor, and its vocal control nuclei differ for size and gene expression of neurotrophic factors. Male Bengalese finches sing complex songs "under indirect sexual selection": in the absence of species recognition, predator avoidance, stress, and other pressures in the wild, they learned a wide-ranging phonology to attract females, "considering the domesticated environment also as a special ecological niche" (Suzuki et al., in this issue). Sexual selection, domestication and reduced stress level, all resulted in a balance between glucocorticoid- and mineralocorticoid receptors in vocal control nuclei and the avian amygdala as well as in the evolution of song complexity, in line with darwinian-fisherian runaway selection for extreme vocal performance. As Darwin notes (1871, chap. XIII, p. 67), "it is not difficult to imagine the steps by which the notes of a bird, primarily used as a mere call or for some other purpose, might have been improved into a melodious love song", although in the case of domestication the "man's taste" could play a relevant role.

\section{Beyond sexual sterotypes}

Sexual selection occurs before, during and after copulation, although the former is perhaps the best-studied. Post-copulatory sexual selection, including sperm competition and cryptic female choice, are now increasingly the subjects of research. Less widely examined, however, are the structures needed for copulation itself, a lack that is addressed by Brennan et al. (in this issue). They examine such structures in two subgroups of the galloanseriform superfamily, Japanese quail and ducks. Like most birds, Japanese quail have a non-intromittent penis, and they have evolved a unique foam gland that produces a meringue-like substance transferred into the female's cloaca during copulation. The secretions of the gland function in post-copulatory sexual selection. Male ducks, in contrast, possess an often elaborate intromittent organ that figures in sexual conflict, with female reproductive tracts apparently having evolved as a "counter-move" to control fertilization. To date, however, researchers have not attempted to examine the neuroendocrine control of these structures as different manifestations of sexual selection, something Brennan and colleagues begin here.

Genes are "fragrant." That is, their gene products or specific ligands smell and may influence mate choice, if these chemosignals are polymorphic enough to, for example, reveal the MHC (major histocompatibility complex) genotype of the partner and hence his or her compatibility in producing offspring with an optimally variable immune response (Vollrath and Milinski, 1995; Milinski, 2006). This topic continues to garner broad interest and generate considerable discussion about the chemical nature of the polimorphic odor signal. Sticklebacks exhibit a two-step mate choice (Milinski, in this issue). First, a female stickleback prefers the scent of a male that offers the optimal complementation of her own alleles. Second, she checks the brighter ones, since the full expression of sex-dimorphic ornament, like the red breeding coloration of males, is dependent on health and vigor (Hamilton and Zuk, 1982). Research may be biased toward modalities that are relatively easily accessible to the human observer. But sexual selection may work via chemical as well as visual stimuli, as comparative experimental evidence on MHC fragrant genes suggest also in humans, "in a never-ending co-evolutionary arms race according to Red Queen dynamics" (Milinski, in this issue). Recent data on neurohormones modulating reproductive and social behavior in the three-spined sticklebacks (Klesczyńska et al., 2012; Kulczykowska and Klesczyńska, 2014) support the relevance of this traditional model organism in behavioral, genetic and neural studies.

Perhaps no topic in sexuality studies attracts as much attention as homosexual behavior in humans, and often the first question to biologists is whether similar behavior occurs in non-humans. Vasey (in this issue) reviews over 20 years of research on female-female sexual behavior in Japanese macaques, noting that such behavior appears to be sexually motivated, rather than as a mechanism for social interaction. This work places sexual behavior in a broader context; not all sexually related activity leads directly to reproduction.

\section{The brain storm of sex differences}

The relationship between non-human and human primates is a longstanding controversial topic. As Darwin pointed out (1871), "the difference in mind between man and the higher animals, great as it is, is it certainly one of degree and not of kind". Recent neurogenomic research, reviewed by Stanyon and Bigoni (in this issue), support both similarities and differences between the groups, as well as the influence of sexual selection. In anthropoid primates, larger brains are associated with monogamous mating systems and lowered mate competition among males (Schillaci, 2006), although both sexes may have armaments and ornaments. In the scenario of "the social brain hypothesis" (Dunbar, 2009), female affiliative sociality emphasizes the neocortex, the mirror neuron activity and the cognitive areas, whereas male competitive sociality is more closely related to the emotional responses of subcortical units and visual-spatial skills. If socialization modulates both neuroanatomy and sex differences, lifelong pair-bonding, high paternal investment and the unique multifamily structure in the human lineage lead to the dramatic expansion of the cerebral cortex and to gender dimorphism along complex networks involved in visual-spatial skills, social cognition and decision-making. Feis et al. (2013) have shown through MR (magnetic resonance) multimodal images that the proportion of grey matter is larger in the female, while the male brain contains more white matter tissue along a gradient from frontal to occipital cortex. In short, in human and non-human primates "a feedback system links behavior, social organization, and gene expression to sexual dimorphism: from the form and function of genitalia to neuroanatomy and the genome" (Stanyon and Bigoni, in this issue).

Gender differences in the capacity of empathy - "the instinct of sympathy" of Darwin (1872, p. 360) in The expression of the emotions in man and animals - is the subject of a comprehensive review by Christov-Moore et al. (in this issue). Phylogenetic and ontogenetic roots of empathy have been compared in human and nonhuman primates of various age and both sexes, beyond any gender stereotype of more empathetic females - the primary caregivers - and less emotional males. The authors explore two categories of empathy across a broad range of behavioral/neural measures and non-invasive techniques. The affective empathy, in which females are very responsive, sharing and "mirroring" the others' emotions and mainly infants' signals, activates frontoparietal, temporal and subcortical areas. The cognitive empathy, a conscious process through which males infer the others' internal states, evaluating and "mentalizing" their intentions, involves the cingulate, prefrontal and temporal regions, i.e. the utilitarian decision-making systems. Empathy is a multilayered process, involving the activity of visuo-motor and audio-visual mirror neurons, beyond further neural mechanisms, due its multimodal nature. The neuroscience 
of empathy (Zaki and Ochsner, 2012) has been reviewed using a Darwinian evolutionary scenario in an evo-devo perspective: sex differences are affected by prenatal androgen exposure and hormone reactivity, begin very early in infants, appear to be stable throughout the life and are influenced by the cultural context. The "ancient biological phenomenon" of empathy (Christov-Moore et al., in this issue) is not a cultural by-product of our socialization but may have evolved in primates in response to different male and female roles.

In humans, facial attractiveness has been much studied as a basis for mate choice and other forms of reproductive behavior. Hahn and Perrett (in this issue) take a neurobiological approach in examining common mechanisms behind reproductive motivation. They find differences in brain activity in response to preferred faces depending on gender and sexual orientation, among other factors. They also note that our understanding of what constitutes an "attractive" face is still not well understood from a neurological standpoint, arguing for studies that combine neuroimaging, genetics, and hormonal patterns. The biological basis of human brain differences in relation to sex and gender (Ball et al., in this issue) is the contentious subject of many popular books: "tempests and tales" (McCarthy and Ball, 2011). If neural systems are involved in the processes of mate choice and social competition for mates in both sexes (CluttonBrock and Huchard, 2013), the brain could be viewed as a secondary sex characteristic, although "ecological" pressures - as underlined by Darwin - and additional drives may shape the phenotype in a sex-specific manner. Following the classical hypothesis of organizational and activational effects of sex steroids, the authors expand their review to birds and mammals, after useful explanations of terms such as sex determination, sex differences and sex dimorphism. There are so many and ubiquitous gender differences in the function and structure of human brain that the authors here adopt the cautious approach indicated by Cahill (2010), i.e. to investigate "why" and "why not" they occur, and "how" may be tested in their proximal and ultimate mechanisms. "In the intervening years two important things have happened. One is that we have expanded our appreciation for sex differences in the brain beyond the realm of reproduction, and second, we have substantially advanced the frontier in terms of identifying the cellular pathways mediating sex differences", which are "unique to each endpoint" (Ball et al., in this issue). Neuroanatomical sex differences with all their complex variants, hormonal and genetic influences, post-natal effects support the marked gender plasticity in human behavior and brain. The interaction between hormones, genes and environment may induce sex differences in the cognitive domain as well as in the juvenile play. In the end, a variety of mechanisms, consistent with adaptive and non-adaptive scenarios, are involved in sex-biased traits inside the evolutionary puzzle of sexual selection.

\section{References}

Ball, G.F., Balthazart, J., McCarthy, M., 2014. Is it useful to view the brain as a secondary sexual characteristic? Neurosci. Biobehav. Rev. (in this issue).

Barske, J., Schlinger, B.A., Wikelski, M., Fusani, L., 2011. Female choice for male motor skills. Proc. R. Soc. B 278, 3523-3528.

Beani, L., Dessì-Fulgheri, F., Cappa, F., Toth, A., 2014. The trap of sex in social insects: from female to male perspective. Neurosci. Biobehav. Rev. (in this issue).

Berglund, A., Bisazza, A., Pilastro, A., 1996. Armaments and ornaments: an evolutionary explanation of traits of dual utility. Biol J Linnean Soc 58, 385-399.

Brennan, P., Stewart, R., Adkins-Regan, E., 2014. Comparing the neuroendocrine mechanisms of primary sexual characters in quail and ducks: the foam gland and the penis. Neurosci. Biobehav. Rev. (in this issue).

Cahill, L., 2010. Sex influences on brain and emotional memory: the burden of proof has shifted. Prog. Brain Res. 186, 29-40.

Chandler, C.H., Ofria, C., Dworkin, I., 2013. Runaway sexual selection leads to good genes. Evolution 67, 110-119.

Christov-Moore, L., Simpson, E.A., Coudé, G., Grigaytile, K., Iacoboni, M., Ferrari, P., 2014. Empathy: gender effects in brain and behavior. Neurosci. Biobehav. Rev. (in this issue).
Clutton-Brock, T., Huchard, E., 2013. Social competition and its consequences in female mammals. Journal of Zoology 289, 151-171.

Darwin, C., 1871. The Descent of Man, and Selection in Relation to Sex. John Murray, London.

Darwin, C., 1872. The Expression of the Emotions in Man and Animals. John Murray London.

Dunbar, R.I., 2009. The social brain hypothesis and its implications for social evolution. Ann. Hum. Biol. 36, 562-572.

Feis, D.L., Brodersen, K.H., von Cramon, D.Y., Luders, E., Tittgemeyer, M., 2013. Decoding gender dimorphism of the human brain using multimodal anatomical and diffusion MRI data. Neuroimage 70, 250-257.

Fisher, R.A., 1958. The Genetical Theory of Natural Selection. Dover Publications New York.

Francis, R.C., 1995. Evolutionary neurobiology. Trends Ecol. Evol. 10, 276-281.

Fusani, L., Barske, J., Day, L.D., Fuxhjanger, M.J., Schlinger, B.A., 2014. Physiological control of elaborate male courtship: female choice for neuromuscular systems. Neurosci. Biobehav. Rev. (in this issue).

Gendron, C.M., Kuo, T.H., Harvanek, Z.M., Chung, B.Y., Yew, J.Y., Dierick, H.A., Pletcher S.D., 2014. Drosophila life span and physiology are modulated by sexual perception and reward. Science 343 (6170), 544-548.

Kokko, H., Brooks, R., McNamara, J.M., Houston, A.I., 2002. The sexual selection continuum. Proc. R. Soc. Lond. B 269, 1333-1340.

Kokko, H., Jennions, M.D., Brooks, R., 2006. Unifying and testing models of sexua selection. Annu. Rev. Ecol. Evol. Syst. 37, 43-66.

Klesczyńska, A., Sokolowska, E., Kulczykowska, E., 2012. Variation in brain arginine vasotocin (AVT) and isotocin (IT) levels with reproductive stage and social status in males of three-spined stickleback (Gasterosteus aculeatus). Gen. Comp. Endocrinol. 175, 290-296.

Kulczykowska, E., Klesczyńska, A., 2014. Brain arginine vasotocin and isotocin in breeding female three-spined stickleback (Gasterosteus aculeatus): the presence of male and egg deposition. Gen. Comp. Endocrinol. 204, 8-12.

Hahn, A., Perrett, D., 2014. Neural and behavioral responses to attractiveness in adult and infant faces. Neurosci. Biobehav. Rev. (in this issue).

Hamilton, W.D., Zuk, M., 1982. Heritable true fitness and bright birds: a role for parasites? Science 218, 384-387.

Herberstein, M., Wignall, A., Hebets, E., Schneider, J., 2014. Dangerous mating systems: signal complexity, signal content and neural capacity in spiders. Neurosci. Biobehav. Rev. (in this issue).

May, P.G., Heulett, S.T., Farrel, T.M., Pilgrim, M.A., 1997. The ecology of pigmy rattlesnakes. Reptil. Amphib. Mag., 36-49.

McCarthy, M., Ball, G.F., 2011. Tempests and tales: challenges in the study of sex differences in the brain. Biol. Sex Differ. 2, 4

Milinski, M., 2014. Arms races, ornaments and fragrant genes: the dilemma of mate choice in fishes. Neurosci. Biobehav. Rev. (in this issue).

Milinski, M., 2006. The major histocompatibility complex, sexual selection, and mate choice. Annu. Rev. Ecol. Evol. Syst. 37, 159-186.

Okanoya, K., 2004. The Bengalese finch: a window on the behavioral neurobiology of birdsong syntax. Ann. N. Y. Acad. Sci. 1016, 724-735.

Owens, P.F., 2006. Where is behavioural ecology going? Trends Ecol. Evol. 21, 356-361.

Prum, R.O., 2012. Aesthetic evolution by mate choice: Darwin's really dangerous idea. Philos. Trans. R. Soc. B 367, 2253-2265.

Stanyon, R., Bigoni, F., 2014. Sexual selection and the evolution of behavior, morphology, neuroanatomy and genes in humans and other primates. Neurosci. Biobehav. Rev. (in this issue).

Reznick, D., 1985. Costs of reproduction: an evaluation of the empirical evidence. Oikos 44, 257-267.

Schillaci, M.A., 2006. Sexual selection and the evolution of brain size in primates PLOS ONE 1, e62.

Suzuki, K., Ikebuchi, M., Bischof, H.J., Okanoya, K., 2014. Behavioral and neural tradeoffs between song complexity and stress reaction in a wild and a domesticated finch strain. Neurosci. Biobehav. Rev. (in this issue).

Vasey, P., 2014. Female homosexual behavior and intersexual mate competition in Japanese macaques: implications for sexual selection theory. Neurosci. Biobehav. Rev. (in this issue).

Vollrath, F., Milinski, M., 1995. Fragrant genes help Damenwahl. Trends Ecol. Evol. 10, 307-308.

West-Eberhard, M.J., 2014. Darwin's forgotten idea: the social essence of sexual selection. Neurosci. Biobehav. Rev. (in this issue).

Zahavi, A., 1975. Mate selection - a selection for handicap. J. Theor. Biol. 53, 205-214

Zaki, J., Ochsner, K.N., 2012. The neuroscience of empathy: progress, pitfalls and promise. Nat. Neurosci. 15 (5), 675-680, http://dx.doi.org/10.1038/nn.3085.

Zuk, M., Wedell, N., 2014. Parasites and pathogens in sexual selection. In: Shuker D.M., Simmons, L.W. (Eds.), The Evolution of Insect Mating Systems. Oxford University Press, pp. 242-253.

Zuk, M., McKean, K.A., 1996. Sex differences in parasite infections: patterns and processes. Int. J. Parasitol. 26, 1009-1024.

Laura Beani

Department of Biology, University of Florence, Italy

Marlene Zuk

Department of Ecology, Evolution and Behavior, University of Minnesota, USA

Available online 7 November 2014 